\title{
Field Representations of the Conformal Group with Continuous Mass Spectrum
}

\author{
W. Rühl \\ Universität Trier-Kaiserslautern, Fachbereich Physik, Kaiserslautern
}

Received December 4, 1972

\begin{abstract}
The discrete series of the conformal group $S U(2,2)$ is realized on a Hilbert space of holomorphic functions over a bounded domain or the field theoretic tube domain. The boundary values of these functions form Hilbert spaces of distributions. For the realization over the tube domain the boundary distributions transform like classical spinorial fields with a continuous mass spectrum extending from zero to infinity The reduction of these field realizations of the whole discrete series into unitary irreducible representations of the inhomogeneous Lorentz group is explicitly given.
\end{abstract}

\section{Summary and Notations}

We are looking for classical fields $\Phi_{x}(x), x$ a vector of Minkowski space, such that for any element $g$ of the conformal group $S U(2,2)$

$$
\left(T_{g} \Phi\right)_{\alpha}(x)=\sum_{\beta} S_{\alpha \beta}(g, x) \Phi_{\beta}\left(g^{-1} x\right)
$$

where $g$ acts on the coordinates $x$ in the familiar fashion (see Eqs. (57b), (60b), (62 b)). We want $T_{g}$ to satisfy the group law

$$
T_{g_{1}} T_{g_{2}}=T_{g_{1} g_{2}} .
$$

Due to (2) the finite dimensional matrix $S$ must satisfy

$$
\sum_{\beta} S_{\alpha \beta}\left(g_{1}, 0\right) S_{\beta \gamma}\left(g_{2}, 0\right)=S_{\gamma \gamma}\left(g_{1} g_{2}, 0\right)
$$

for any pair $g_{1}, g_{2}$ of elements of the conformal group that leave the point $x=0$ unchanged. These group elements form the stabilizer subgroup for $x=0$. For convenience of notation we identify the conformal group with the group $S U(2,2)$. Then the stabilizer consists of the product of

a) the homogeneous Lorentz group $S L(2, C)$;

b) the subgroup of dilations $D$;

c) the group of special conformal transformations that has the structure of an abelian translation group in four real dimensions:

d) two elements of the centre $Z_{4}$ of $S U(2,2)$ that are not yet contained in $S L(2, C)$. 
The stabilizer has the structure

$$
G=Z_{4} \otimes\left\{\left(S L(2, C) / Z_{2} \otimes D\right) \times T_{4}\right\}
$$

where $\otimes$ denotes the direct and $\times$ the semidirect product.

If in turn a matrix representation of the stabilizer $G$ is given, then the general matrix $S(g, x)$ can be reconstructed by the theory of induced representations [1]. In the field representations we are going to construct, the special conformal transformations act trivially on the field at $x=0$. The matrices $S(g, 0)$ present therefore an irreducible representation of the direct product of $S L(2, C), D$, and $Z_{4}$. This implies that the fields can be given in a spinorial basis $\Phi_{A \dot{B}}(x),-j_{1} \leqq A \leqq j_{1},-j_{2} \leqq B \leqq j_{2}$, for the representation $\left(j_{1}, j_{2}\right)$ of $S L(2, C)$. The dimension $d$ of the field (see (58), $d=-l$ in the notation of Ref. [1]) is such that $n=d+j_{1}+j_{2}$ is an integer for the discrete series. Projective representations of the conformal group or unitary representations of the infinitesimal conformal group are obtained if we interpolate the discrete series, that is to say, if we let $n$ assume non-integral values.

Graev [2] has defined the discrete series of $S U(2,2)$ and we use his work as a starting point for our investigation. Further results have been obtained by Yao [3]. Graev constructs the discrete series by means of Hilbert spaces of holomorphic functions over a bounded domain. To illustrate the different realizations it is helpful to remember the discrete series representations of $S U(1,1)$ that are quite analogous. The realization by holomorphic functions $f(z)$ over the unit circle with the Hilbert space norm

$$
\|f\|_{k}^{2}=\frac{2 k-1}{\pi} \int_{|z|<1}|f(z)|^{2}\left(1-|z|^{2}\right)^{2 k-2}|d z|
$$

was historically the first studied [4]. By a straightforward analytic one-to-one map one obtains a realization by means of holomorphic functions over the upper half $w$-plane. These holomorphic functions $F(w)$ possess boundary values $\Phi(x)$ on the real axis that are assumed on the boundary in the sense of a distribution theoretic limit and are themselves distributions [5]. In turn we can extend these distributions $\Phi(x)$ into the upper half plane by a Hilbert transform. They are therefore in a one-to-one relation with the holomorphic functions $F(w)$. This yields a third realization of the discrete series by means of Hilbert spaces of distributions $\Phi(x)$. These are the "field representations". Finally we apply a Fourier transformation to $\Phi(x)$ and obtain a distribution $\Psi(p)$ with support on the positive real axis. This is a fourth realization for the discrete series that is very comfortable for the purpose of reducing the representations.

In the case of $S U(2,2)$ the situation is analogous. We start from Graev's realization on the Hilbert space of holomorphic functions over 
the bounded domain $\mathbb{D}$, the "generalized unit circle". By means of an analytic one-to-one map we obtain holomorphic functions $F(W)$ over the "generalized upper half plane" that is identical with the field theoretic tube domain $\mathrm{T}$ :

$$
\mathbb{T}=\left\{\begin{array}{l}
w_{\mu}=x_{\mu}+i y_{\mu}, \quad x \text { and } y \text { real. } \\
y \in L_{+}, \text {that is } y_{0}>\left(\sum_{h} y_{h}^{2}\right)^{1 / 2}
\end{array}\right\} .
$$

The boundary distributions are taken on the Shilov boundary of $\mathbb{T}$, the real Minkowski space. They are spinorial fields $\Phi_{A \dot{B}}(x)$, whose Fourier transforms $\Psi_{A \dot{B}}(p)$ have support only over the forward light cone $L_{+}$. These fields $\Psi_{A \dot{B}}(p)$ help us to solve the problem of decomposing the representations of the discrete series of $S U(2,2)$ into irreducible representations of the inhomogeneous Lorentz group. We need only keep $p$ on a mass shell $p^{2}=m^{2}$ and reduce the spinorial representation of $S L(2, C)$ with respect to $S U(2)$. It is obvious that a mass spectrum $0<m^{2}<\infty$ results and that this is a consequence of the analyticity of the functions $F(W)$ over the tube domain.

Finally we can map the Hilbert space of distributions $\Phi_{A \dot{B}}(x)$ isomorphically on the Hilbert subspace of vectors

$$
\hat{\Phi}_{A \dot{B}}(x)|0\rangle
$$

where $\hat{\Phi}_{A \dot{B}}(x)$ is a Greenberg type generalized free field operator and $|0\rangle$ is the vacuum state. The Lehmann weight function for this field is a homogeneous function of the mass [6].

\section{Classical Spinorial Fields}

In this section we present a recollection of formulas on spinorial fields that we need in the sequel. If we require covariance (1) of a field with respect to the inhomogeneous Lorentz group only, the stabilizer reduces to the homogeneous Lorentz group. Let $a$ denote a matrix of $S L(2, C)$, $\left(j_{1}, j_{2}\right)$ an irreducible spinorial representation of $S L(2, C)$. We make use of the known rotation functions $D_{q_{1} q_{2}}^{j}$ holomorphically extended from $S U(2)$ to $S L(2, C)$ :

$$
\begin{aligned}
D_{q_{1} q_{2}}^{j}(a)= & {\left[\left(j+q_{1}\right) !\left(j-q_{1}\right) !\left(j+q_{2}\right) !\left(j-q_{2}\right) !\right]^{\frac{1}{2}} } \\
& \cdot \sum_{n}\left[n !\left(j+q_{1}-n\right) !\left(j+q_{2}-n\right) !\left(n-q_{1}-q_{2}\right) !\right]^{-1} \\
& \cdot a_{11}^{n} a_{12}^{j+q_{1}-n} a_{21}^{j+q_{2}-n} a_{22}^{n-q_{1}-q_{2}} .
\end{aligned}
$$


They are homogeneous polynomials of degree $2 j$ in the matrix elements of $a$. A spinorial field transforms then as

$$
\left(T_{a} \Phi\right)_{A \dot{B}}(X)=\sum_{C D} D_{A C}^{i_{1}}(a) \Phi_{C \dot{D}}\left(a^{-1} X a^{-1, \dagger}\right) D_{D B}^{j_{2}}\left(a^{\dagger}\right)
$$

under a homogeneous Lorentz transformation. $X$ denotes a hermitean $2 \times 2$ matrix that is obtained from the four-vector $x$ by

$$
X=x_{0} E+\sum_{k=1}^{3} x_{k} \sigma_{k}
$$

Similarly we define $\hat{X}$ by

$$
\hat{X}=x_{0} E-\sum_{k=1}^{3} x_{k} \sigma_{k}
$$

and corresponding matrices for other four-vectors. It is obvious that (7) simplifies if we use a matrix notation for the spinorial field. Using it we write

$$
\left(T_{a} \Phi\right)(X)=D^{j_{1}}(a) \Phi\left(a^{-1} X a^{-1 . \dagger}\right) D^{j_{2}}\left(a^{\dagger}\right) .
$$

This notation is throughout used in the sequel.

We assume now that $\Phi(x)$ satisfies the Klein-Gordon equation

$$
\left(\square_{x}+m^{2}\right) \Phi(x)=0, m^{2}>0 .
$$

A Fourier transformation

$$
\Phi(x)=(2 \pi)^{-\frac{3}{2}} \int d^{4} p \delta\left(p^{2}-m^{2}\right) e^{i p x} \Psi(p)=\mathscr{F}(\Psi)
$$

allows us to split $\Phi(x)$ into positive and negative frequency parts

$$
\begin{gathered}
\Psi(p)=\Psi^{(+)}(p)+\Psi^{(-)}(p), \Psi^{(+)}(p)=\theta\left(p_{0}\right) \Psi(p) \\
\Phi^{( \pm)}(x)=\mathscr{F}\left(\Psi^{( \pm)}\right)(x) .
\end{gathered}
$$

From the positive frequency parts we build a Hilbert space with the scalar product

$$
\begin{aligned}
\left(\Phi_{1}^{(+)}, \Phi_{2}^{(+)}\right)= & \int d^{4} p \delta\left(p^{2}-m^{2}\right) \\
& \cdot \operatorname{Tr}\left\{\left(\Psi_{1}^{(+)}(P)\right)^{\dagger} D^{j_{1}}\left(\frac{\hat{P}}{m}\right) \Psi_{2}^{(+)}(P) D^{j_{2}}\left(\frac{\hat{P}}{m}\right)\right\}
\end{aligned}
$$

$P$ and $\hat{P}$ are defined through $p$ like $X$ and $\hat{X}$ through $x$, see (8) and (9). The adjoint symbol refers to the spinor matrix form of $\Psi$. This scalar product is obviously invariant. One needs only take into account that the substitution of $P$ by $a P a^{\dagger}$ entails the substitution of $\hat{P}$ by $\left(a^{\dagger}\right)^{-1} \hat{P} a^{-1}$, as follows from $P \hat{P}=m^{2} E$. Finally we notice that $\Phi^{(+)}(x)$ is the boundary 
value of a holomorphic function in the tube domain (5), provided it belongs to some class of distributions.

The connection of $\Phi(x)$ and $\Psi(p)$ with irreducible unitary representations of the inhomogeneous Lorentz group is easily established by the transformation

$$
\Psi^{(+)}(P)=D^{j_{1}}\left(\left(\frac{P}{m}\right)^{\frac{1}{2}}\right) \tilde{\Psi}^{(+)}(P) D^{j_{2}}\left(\left(\frac{P}{m}\right)^{\frac{1}{2}}\right)
$$

where the square roots are defined to be positive-definite hermitean. Then the scalar products reduce to

$$
\left(\Phi_{1}^{(+)}, \Phi_{2}^{(+)}\right)=\int d^{4} p \delta\left(p^{2}-m^{2}\right) \operatorname{Tr}\left\{\tilde{\Psi}^{(+)}(P)^{\dagger} \tilde{\Psi}^{(+)}(P)\right\} .
$$

Under transformations of the homogeneous Lorentz group we have

$$
T_{a} \tilde{\Psi}^{(+)}(P)=D^{j_{1}}(u) \tilde{\Psi}^{(+)}\left(P^{\prime}\right) D^{j_{2}}\left(u^{+}\right)
$$

with

$$
\begin{aligned}
P^{\prime} & =a^{-1} P a^{-1, \dagger}, \\
u & =\left(\frac{P}{m}\right)^{-\frac{1}{2}} a\left(\frac{P^{\prime}}{m}\right)^{+\frac{1}{2}} .
\end{aligned}
$$

The matrix $u$ can easily be shown to be unitary. It is called Wigner's rotation. By means of the relation

$$
D_{q_{1} q_{2}}^{j}\left(u^{\dagger}\right)=(-1)^{q_{1}-q_{2}} D_{-q_{2},-q_{1}}^{j}(u)
$$

for the functions (6) and vector coupling coefficients (following the conventions of Edmonds [7]) we get

$$
\tilde{\tilde{\Psi}}_{Q}^{y}(P)=\sum_{q q^{\prime}}(-1)^{j_{2}+q^{\prime}}\left(J, Q \mid j_{1}, q ; j_{2},-q^{\prime}\right) \tilde{\Psi}_{q q^{\prime}}^{(+)}(P) \text {. }
$$

Inserting this into (17) it results

$$
\left(T_{a} \tilde{\tilde{\Psi}}\right)_{Q}^{y}(P)=\sum_{Q^{\prime}} D_{Q Q^{\prime}}^{y}(u) \tilde{\tilde{\Psi}}_{Q^{\prime}}^{y}\left(P^{\prime}\right)
$$

Thus the spinorial fields have been decomposed into a sum of "Wigner" fields, that correspond to irreducible unitary representations of the inhomogeneous Lorentz group. The spin $J$ runs from $\left|j_{1}-j_{2}\right|$ till $j_{1}+j_{2}$.

\section{Graev's Realization of the Discrete Series}

We define a linear space of functions $f\left(\Xi_{1}, \Xi_{2}, Z\right)$ whose three arguments are complex two-by-two matrices. The function $f$ is assumed to be a homogeneous polynomial of degree $2 j_{1}\left(2 j_{2}\right)$ in the elements of the first column of $\Xi_{1}\left(\Xi_{2}\right)$. For fixed $\Xi_{1}$ and $\Xi_{2}$ it is a holomorphic 
function of the four elements of $Z$ in the domain $\mathbb{D}$ that is defined by the constraint of positive definiteness

$$
E-Z^{\dagger} Z>0
$$

In addition we introduce a norm and a corresponding scalar product by

$$
\begin{gathered}
\|f\|_{n}^{2}=c_{n j_{1} j_{2}} \int_{\mathbb{D}}\left|f\left(\overline{N_{1} K_{1}}, N_{2} K_{2}, Z\right)\right|^{2}\left[\operatorname{det}\left(E-Z^{\dagger} Z\right)\right]^{n-4} \\
\cdot d \mu\left(K_{1}\right) d \mu\left(K_{2}\right)|d Z| .
\end{gathered}
$$

In this expression the notations mean

$$
\begin{aligned}
N_{1} & =\left(E-Z Z^{\dagger}\right)^{-\frac{1}{2}} \\
N_{2} & =\left(E-Z^{\dagger} Z\right)^{-\frac{1}{2}} \\
|d Z| & =\prod_{i, j} d\left(\operatorname{Re} z_{i j}\right) d\left(\operatorname{Im} z_{i j}\right)
\end{aligned}
$$

$K_{1,2}$ are unitary matrices, $d \mu(K)$ is the normalized Haar measure on $U(2)$.

For the norm (23) to be finite, the functions $f$ cannot increase arbitrarily at the boundary of $\mathbb{D}$. The increase can in fact be estimated by the Bergman kernel as we shall show later. From the theory of Hilbert spaces of holomorphic functions [8] one knows that functions $f$ holomorphic in $\mathbb{D}$ with a finite norm (23) form a Hilbert space. We denote it by $\mathscr{A}_{n j_{1} j_{2}}^{2}(\mathbb{D})$. The real number $n$ must be such that the norm (23) exists at least for the constant functions. This yields the restriction

$$
n>2 j_{1}+2 j_{2}+3 \text {. }
$$

Lateron we shall investigate the possibility of extending in $n$ to lower values. Finally we mention that we can define Hilbert spaces of antiholomorphic functions over $\mathbb{D}$ that carry the second branch of the discrete series. Because of the analogous properties of both branches we restrict our discussion to holomorphic functions. The two branches of the discrete series are conjugate to each other.

A matrix $M$ of $S U(2,2)$ satisfies the two constraints

where

$$
M^{-1} H=H M^{\dagger}, \operatorname{det} M=1
$$

$$
H=\left(\begin{array}{cc}
-E & 0 \\
0 & E
\end{array}\right)
$$

Each matrix $M$ can be given in a form of four two-by-two submatrices $A, B, C, D$

$$
M^{-1}=\left(\begin{array}{ll}
A & B \\
C & D
\end{array}\right)
$$


that have properties following from (25). For these and more details see [9] where the case $j_{1}=j_{2}=0$ has been studied extensively. Each matrix $M$ defines an analytic automorphism of $\mathbb{D}$ by

$$
\begin{aligned}
Z \rightarrow Z^{\prime} & =(A Z+B)(C Z+D)^{-1} \\
& =\left(A^{\dagger}+Z B^{\dagger}\right)^{-1}\left(C^{\dagger}+Z D^{\dagger}\right) .
\end{aligned}
$$

These automorphisms themselves form a group with the structure $S U(2,2) / Z_{4}$. With the help of these automorphisms we define unitary operators in $\mathscr{A}_{n j_{1} j_{2}}^{2}(\mathbb{D})$ by

$$
\begin{aligned}
& T_{M} f\left(\Xi_{1}, \Xi_{2}, Z\right) \\
& =\operatorname{det}(C Z+D)^{-n} f\left(\overline{\left(A+B Z^{\dagger}\right)} \Xi_{1},(C Z+D) \Xi_{2}, Z^{\prime}\right) .
\end{aligned}
$$

The unitarity of $T_{M}$ can be proved by elementary algebra. These operators $T_{M}$ form a unitary irreducible representation for $S U(2,2)$ if $n$ is an integer and a projective representation if $n$ is non-integral. In fact, $\operatorname{det}(C Z+D)$ is always nonzero in $\mathbb{D}$. But due to the multiple connectedness of $S U(2,2)$ we can find a closed path on $S U(2,2)$ such that $\operatorname{det}(C Z+D)$ encircles zero in the complex plane. One such path is given for example by

$$
A=\left(\begin{array}{ll}
e^{i \varphi} & 0 \\
0 & 1
\end{array}\right)=D^{-1}, B=C=0,0 \leqq \varphi \leqq 2 \pi .
$$

In Hilbert spaces of holomorphic functions the linear functional $Z_{\Xi_{1} \Xi_{2}}$ :

$$
Z_{\Xi_{1} \Xi_{2}}(f)=f\left(\Xi_{1}, \Xi_{2}, Z\right), \quad Z \in \mathbb{D}
$$

is bounded and can therefore be represented as a scalar product

$$
f\left(\Xi_{1}, \Xi_{2}, Z\right)=\left(K_{\Xi_{1}, \Xi_{2}, Z}, f\right)_{n}
$$

where $K$, the Bergman kernel, is an element of $\mathscr{A}_{n j_{1} j_{2}}^{2}(\mathbb{D})$ for fixed $\Xi_{1}, \Xi_{2}$, and $Z$. Its covariance under the unitary transformations (29) allows us to determine it up to a normalization constant $c_{B}$

$$
\begin{aligned}
K_{\Xi_{1}, \Xi_{2}, Z}\left(\Xi_{1}^{\prime}, \Xi_{2}^{\prime}, Z^{\prime}\right)=c_{B} D_{j_{1} j_{1}}^{J_{1}}\left(\Xi_{1}^{\prime T}\left(E-Z^{\prime} Z^{\dagger}\right) \bar{\Xi}_{1}\right) \\
\cdot D_{j_{2} j_{2}}^{j_{2}}\left(\Xi_{2}^{\dagger}\left(E-Z^{\dagger} Z^{\prime}\right) \Xi_{2}^{\prime}\right) \operatorname{det}\left(E-Z^{\dagger} Z^{\prime}\right)^{-n} .
\end{aligned}
$$

Both constants $c_{B}$ and $c_{n j_{1} j_{2}}(23)$ are connected by

$$
\begin{aligned}
c_{B} c_{n j_{1} j_{2}}=\pi^{-4}\left(2 j_{1}+1\right) & \left(2 j_{2}+1\right)(n-1)\left(n-2 j_{1}-2\right) \\
& \cdot\left(n-2 j_{2}-2\right)\left(n-2 j_{1}-2 j_{2}-3\right) .
\end{aligned}
$$

If we require that for the special function

$$
f\left(\Xi_{1}, \Xi_{2}, Z\right)=D_{j_{1} j_{1}}^{j_{1}}\left(\Xi_{1}\right) D_{j_{2} j_{2}}^{j_{2}}\left(\Xi_{2}\right)
$$


the norm is one, then $c_{B}$ has to be chosen equal one and $c_{n j_{1} j_{2}}$ equals the right hand side of (32). From Schwarz's inequality we have

$$
\left|f\left(\Xi_{1}, \Xi_{2}, Z\right)\right| \leqq\left\|K_{\Xi_{1}, \Xi_{2}, Z}\right\|_{n}\|f\|_{n} .
$$

Since (30) implies

$$
\left\|K_{\Xi_{1}, \Xi_{2}, Z}\right\|_{n}^{2}=K_{\Xi_{1}, \Xi_{2}, Z}\left(\Xi_{1}, \Xi_{2}, Z\right)
$$

it results

$$
\left|f\left(\Xi_{1}, \Xi_{2}, Z\right)\right| \leqq K_{\Xi_{1}, \Xi_{2} . Z}\left(\Xi_{1}, \Xi_{2}, Z\right)^{\frac{1}{2}}\|f\|_{n} .
$$

This is the desired estimate of the increase of the functions of $\mathscr{A}_{n j_{1} j_{2}}^{2}(\mathbb{D})$ at the boundary of $\mathbb{D}$.

The at most polynomial increase of $f$ at the boundary of $\mathbb{D}(36)$ tells us that on the Shilov boundary of $\mathbb{D}$ (that consists of unitary matrices $Z$ ) $f$ tends to a distribution boundary value in a distribution theoretic sense. In the case $j_{1}=j_{2}=0$ we were able to characterize the boundary values [5] as weak derivatives (for $n$ even)

$\Phi(Z)=\left[(\operatorname{det} Z)\left(\frac{\partial}{\partial z_{11}} \frac{\partial}{\partial z_{22}}-\frac{\partial}{\partial z_{12}} \frac{\partial}{\partial z_{21}}\right)+\sum_{i j} z_{i j} \frac{\partial}{\partial z_{i j}}+2\right]^{\frac{n-2}{2}} G(Z)$

where $G(Z)$ is square integrable on the Shilov boundary with respect to the Haar measure on $U(2)$. The method used for the proof rested on Fourier expansions on $U(2)[5,9]$. We shall rederive an analogous result by another method in this article.

We consider next the problem of how to construct a basis in the representation space $\mathscr{A}_{n j_{1} j_{2}}^{2}(\mathbb{D})$ that reduces the representation of $S U(2,2)$ considered if the group is restricted to the maximal compact subgroup. We start by noting that the polynomials

$$
D_{q j}^{j}(\Xi)=D_{j q}^{j}\left(\Xi^{T}\right)
$$

form a basis in the space of homogeneous polynomials of degree $2 j$ in the elements of the first column of $\Xi$. We expand $f\left(\Xi_{1}, \Xi_{2}, Z\right)$ in this basis and obtain in matrix notation

$$
f\left(\Xi_{1}, \Xi_{2}, Z\right)=\left(D^{j_{1}}\left(\Xi_{1}^{T}\right) f(Z) D^{j_{2}}\left(\Xi_{2}\right)\right)_{j_{1} j_{2}} .
$$

Each element of the $\left(2 j_{1}+1\right) \times\left(2 j_{2}+1\right)$ matrix $f(Z)$ is a holomorphic function over $\mathbb{D}$. Operating on $f(Z)$ with the unitary operator $T_{M}$ gives

$$
T_{M} f(Z)=\operatorname{det}(C Z+D)^{-n} D^{j_{1}}\left(A^{\dagger}+Z B^{\dagger}\right) \cdot f\left(Z^{\prime}\right) D^{j_{2}}(C Z+D)
$$

with $Z^{\prime}$ as in (28). The norm assumes the form

$$
\begin{aligned}
\|f\|_{n}^{2}= & c_{n j_{1} j_{2}}\left(2 j_{1}+1\right)^{-1}\left(2 j_{2}+1\right)^{-1} \\
& \cdot \int \operatorname{Tr}\left\{f(Z)^{\dagger} D^{j_{1}}\left(N_{1}^{2}\right) f(Z) D^{j_{2}}\left(N_{2}^{2}\right)\right\} \operatorname{det}\left(E-Z^{\dagger} Z\right)^{n-4}|d Z| .
\end{aligned}
$$


Each function holomorphic over $\mathbb{D}$, in particular each matrix element of $f(Z)$, can be expanded in the homogeneous polynomials

$$
\begin{gathered}
(\operatorname{det} Z)^{m} D_{q_{1} q_{2}}^{j}(Z) \\
m=0,1,2, \ldots \quad 2 j=0,1,2, \ldots \quad-j \leqq q_{1,2} \leqq+j
\end{gathered}
$$

in a series that converges absolutely uniformly on compact subsets of $\mathbb{D}$ $[5,9]$. However, it can be shown by direct computation that the functions

$$
\Delta_{q_{1} q_{2} s_{1} s_{2}}^{j m}\left(\Xi_{1}, \Xi_{2}, Z\right)=D_{j_{1} q_{1}}^{j_{1}}\left(\Xi_{1}^{T}\right) D_{q_{2} j_{2}}^{j_{2}}\left(\Xi_{2}\right)(\operatorname{det} Z)^{m} D_{s_{1} s_{2}}^{j}(Z)
$$

do not form an orthogonal set. The orthogonality can be improved if we define new polynomials by means of vector coupling coefficients [7]

$$
\begin{aligned}
& \Delta_{Q_{1} Q_{2}}^{j m J_{1} J_{2}}\left(\Xi_{1}, \Xi_{2}, Z\right) \\
& =\sum_{q_{1} q_{2} s_{1} s_{2}}(-1)^{j_{1}-q_{1}+j_{2}-q_{2}}\left(j, s_{1} ; j_{1},-q_{1} \mid J_{1}, Q_{1}\right)\left(j, s_{2} ; j_{2},-q_{2} \mid J_{2}, Q_{2}\right) \\
& \cdot \Delta_{q_{1} q_{2} s_{1} s_{2}}^{j m}\left(\Xi_{1}, \Xi_{2}, Z\right) .
\end{aligned}
$$

To understand this result we consider the behaviour of these functions (44) under transformations $T_{M}$ belonging to the maximal compact subgroup of $S U(2,2)$. An element $M$ belongs to the maximal compact subgroup if it has the form

with

$$
M^{-1}=\left(\begin{array}{ll}
K_{1} & 0 \\
0 & K_{2}
\end{array}\right)
$$

$$
\begin{gathered}
K_{1}=e^{i \frac{\varphi}{2}} u_{1}, K_{2}=e^{-i \frac{\varphi}{2}} u_{2} \\
u_{1,2} \in S U(2) .
\end{gathered}
$$

Inserting this into (29) yields

$$
\begin{gathered}
T_{M} \Delta_{Q_{1} Q_{2}}^{j m J_{1} J_{2}}\left(\Xi_{1}, \Xi_{2}, Z\right)=e^{l \varphi\left(N+n-j_{1}-j_{2}\right)} \\
\cdot\left[D^{I_{1}}\left(u_{1}\right) \Delta^{j m I_{1} J_{2}}\left(\Xi_{1}, \Xi_{2}, Z\right) D^{J_{2}}\left(u_{2}\right)\right]_{Q_{1} Q_{2}}
\end{gathered}
$$

where $N=2 j+2 m$. The basis elements (44) with fixed $j, m, J_{1}, J_{2}$ span therefore a basis for an irreducible unitary representation of the maximal compact subgroup. This representation is characterized by the three labels $N, J_{1}, J_{2}$. In turn these three labels do not fix the representation of the maximal compact subgroup uniquely. For given $N, J_{1}, J_{2}$ we may let $j$ still vary over (in integral steps) $\frac{1}{2} \leqq j \leqq \frac{1}{2} N$ (for $N$ odd) $0 \leqq j \leqq \frac{1}{2} N$ (for $N$ even) and

$$
\begin{aligned}
& \left|J_{1}-j_{1}\right| \leqq j \leqq J_{1}+j_{1}, \\
& \left|J_{2}-j_{2}\right| \leqq j \leqq J_{2}+j_{2} .
\end{aligned}
$$


The multiplicity can easily be computed from these constraints. The multiplicity is never bigger than one only if either $j_{1}$ or $j_{2}$ vanishes. Yao [3] calls the case $j_{1} j_{2} \neq 0$ "non-degenerate" as compared with the "degenerate" case when $j_{1} j_{2}=0$.

If we compute the scalar product for two elements of the basis (44) we find as we expect

$$
\begin{aligned}
& \left(\Delta_{Q_{1} Q_{2}}^{j m J_{1} J_{2}^{\prime}}, \Delta_{Q_{1}^{\prime} Q_{2}^{\prime}}^{j^{\prime} J_{1}^{\prime} J_{2}^{\prime}}\right)=\delta_{N N^{\prime}} \delta_{I_{1} J_{1}^{\prime}} \delta_{J_{2} I_{2}^{\prime}} \delta_{Q_{1} Q_{1}^{\prime}} \delta_{Q_{2} Q_{2}^{\prime}} \\
& \text { - } M_{j j^{\prime}}^{N y_{1} J_{2}}\left(n, j_{1}, j_{2}\right) \text {. }
\end{aligned}
$$

In the non-degenerate case the matrix $M$ (with respect to the subscripts) is a certain positive definite matrix that reduces to a single number in the degenerate case. Yao has proposed a method of diagonalizing it (the matrix form derived from the scalar product (48) is a terribly complicated looking object): He has defined an operator $F_{3}$ of the enveloping algebra of $S U(2,2)$ that commutes with the generators of the maximal compact subgroup. It is a third order differential operator in our function space. The diagonalization of this operator (or another similar one) is, however, a hitherto unsolved problem.

\section{The Tube Domain}

The domain $\mathbb{D}(22)$ can be mapped one-to-one on the tube domain $\mathbb{T}$ (5) by the Cayley transformation

$$
\begin{aligned}
& W=i(E-Z)(E+Z)^{-1}=W(Z) \\
& Z=(E-i W)^{-1}(E+i W)=Z(W) .
\end{aligned}
$$

The Hilbert space $\mathscr{A}_{n j_{1} j_{2}}^{2}(\mathbb{D})$ is correspondingly mapped isomorphically on a space $\mathscr{A}_{n_{1} j_{2}}^{2}(\mathrm{~T})$ of holomorphic functions $F(W)$ over $\mathrm{T}$ by $\left(f \in \mathscr{A}_{n_{j_{1} j_{2}}}^{2}(\mathbb{D})\right)$

$$
\begin{gathered}
F(W)=m(W) D^{j_{1}}(E-i W) f(Z(W)) D^{j_{2}}(E-i W) \\
m(W)=2^{2(d-1)}[\operatorname{det}(E-i W)]^{-n}
\end{gathered}
$$

with the norm

where

$$
\begin{aligned}
\|F\|_{n}^{2}= & \frac{c_{n j_{1} j_{2}}}{\left(2 j_{1}+1\right)\left(2 j_{2}+1\right)} \int_{\mathrm{T}} \operatorname{Tr}\left\{F(W)^{\dagger} D^{j_{1}}(\hat{Y}) F(W) D^{j_{2}}(\hat{Y})\right\} \\
& \cdot\left(y^{2}\right)^{n-2 j_{1}-2 j_{2}-4} d^{4} x d^{4} y
\end{aligned}
$$

$$
\begin{gathered}
W=w_{0} E+\sum_{k=1}^{3} w_{k} \sigma_{k} \\
w_{\mu}=x_{\mu}+i y_{\mu}, \quad W=X+i Y,
\end{gathered}
$$


and $Y, \hat{Y}$ are defined by means of the vector $y$ as in (8), (9). By the mapping (49) the Shilov boundary of $\mathbb{D}$ (that consists of unitary matrices) appears as the closure of the image of the real Minkowski space, therefore as one of its possible compactifications.

The operators $T_{M}(40)$ induce analogous operators in the Hilbert space $\mathscr{A}_{n j_{1} j_{2}}^{2}(\mathrm{~T})$ by means of $(50)$

$T_{M} F(W)=[\operatorname{det}(T W+Q)]^{-n} D^{j_{1}}\left(W T^{\dagger}+Q^{\dagger}\right) \cdot F\left(W^{\prime}\right) D^{j_{2}}(T W+Q)$

where

$$
\begin{aligned}
W^{\prime} & =(R W+S)(T W+Q)^{-1} \\
& =\left(W T^{\dagger}+Q^{\dagger}\right)^{-1}\left(W R^{\dagger}+S^{\dagger}\right) .
\end{aligned}
$$

The matrices $R, S, T, Q$ are defined by (see Ref. [9], Eq. (2.16))

$$
\begin{gathered}
M^{-1}=\left(\begin{array}{ll}
A & B \\
C & D
\end{array}\right) \rightarrow \check{M}^{-1}=\left(\begin{array}{rr}
R, & i S \\
-i T, & Q
\end{array}\right)=U\left(\begin{array}{ll}
A & B \\
C & D
\end{array}\right) U^{-1} \\
U=\frac{1}{\sqrt{2}}\left(\begin{array}{rr}
E, & -E \\
E, & E
\end{array}\right) .
\end{gathered}
$$

They satisfy constraints (Ref. [9], Eq. (2.17)) that we will not repeat here. The matrices $\breve{M}$ form a group that can be looked upon as another realization of $S U(2,2)$.

We evaluate now the transformation law (53) for the following subgroups: the group of dilations, the inhomogeneous Lorentz group, and the group of special conformal transformations. The subgroup of dilations consists of the matrices

We obtain from (53)

$$
\check{M}(\lambda)=\left(\begin{array}{ll}
\lambda & 0 \\
0 & \lambda^{-1}
\end{array}\right), \quad \lambda>0 .
$$

$$
\begin{aligned}
T_{M} F(W) & =\lambda^{-2 n+2 j_{1}+2 \jmath_{2}} F\left(W^{\prime}\right) \\
W^{\prime} & =\lambda^{-2} W
\end{aligned}
$$

so that the mass dimension $d$ of our representation is

$$
d=n-j_{1}-j_{2} .
$$

The inhomogeneous Lorentz group consists of the matrices

$$
\begin{array}{r}
\check{M}(a, v)=\left(\begin{array}{cc}
a, & i V a^{-1, \dagger} \\
0, & a^{-1, \dagger}
\end{array}\right) \\
a \in S L(2, C) \\
V=v_{0} E+\sum_{k=1}^{3} v_{k} \sigma_{k}, v_{\mu} \text { real. }
\end{array}
$$


This gives

$$
\begin{aligned}
T_{M} F(W) & =D^{j_{1}}(a) F\left(W^{\prime}\right) D^{j_{2}}\left(a^{\dagger}\right) \\
W^{\prime} & =a^{-1}(W-V) a^{-1, \dagger} .
\end{aligned}
$$

Finally we have the special conformal transformations

$$
\begin{gathered}
\check{M}(u)=\left(\begin{array}{ll}
E & 0 \\
i \hat{U} & E
\end{array}\right) \\
\hat{U}=u_{0} E-\sum_{k=1}^{3} u_{k} \sigma_{k}, u_{\mu} \text { real }
\end{gathered}
$$

that lead to

$$
\left.\begin{array}{c}
T_{M} F(W)=\sigma(u, w)^{-n} D^{j_{1}}(E+W \hat{U}) F\left(W^{\prime}\right) D^{j_{2}}(E+\hat{U} W) \\
w_{\mu}^{\prime}=\sigma(u, w)^{-1}\left[w_{\mu}+w^{2} u_{\mu}\right] \\
\sigma(u, w)=1+2 u_{\mu} w^{\mu}+u^{2} w^{2} .
\end{array}\right\}
$$

If we now go to the boundary $Y \rightarrow 0$, the holomorphic functions $F(W)$ approach their boundary distributions $\Phi(X)$ in a distribution theoretic sense. These distributions $\Phi(X)$ are the field representations of the conformal group. They transform like (57), (60), (62). At the point $W=X=0$ the transformation (62) reduces to the identity

$$
T_{M} F(0)=F(0) .
$$

This proves that the special conformal subgroup of the stabilizer $G$ is represented trivially in our field representations. In the notation of Mack and Salam [1] our representations belong to their class Ia.

\section{Reduction into Irreducible Unitary Representations of the Inhomogeneous Lorentz Group}

We apply a Fourier-Laplace transformation

$$
F(W)=(2 \pi)^{-2} \int_{L_{+}} d^{4} p e^{\imath w p} \Psi(P), w p=w_{\mu} p^{\mu}
$$

( $L_{+}$the forward light cone, see (5)) that maps the Hilbert space of holomorphic functions $\mathscr{A}_{n j_{1} j_{2}}^{2}(T)$ on a Hilbert space of distributions with support on the forward light cone. These distributions are tempered. We are not interested here in the transformations induced by the operators $T_{M}$ on the space of distributions $\Psi(P)$ but rather in the expression of the norm $\|F\|_{n}(51)$ in terms of $\Psi(P)$. It gives us the direct integral decomposition of the representation. 
Inserting (64) into (51), the integration over $x$ yields a delta function and one integration over $p$ is correspondingly trivial. The integral over $y$ is elementary and we obtain

where

$$
\begin{gathered}
\|F\|_{n}^{2}=c \int_{L_{+}} d^{4} p \operatorname{Tr}\left\{\Psi(P)^{\dagger} D^{j_{1}}(-\partial) \Psi(P) D^{j_{2}}(-\partial)\right\} \\
\cdot\left(p^{2}\right)^{-n+2 j_{1}+2 j_{2}+2}
\end{gathered}
$$

$$
\delta=E \frac{\partial}{\partial p_{0}}+\sum_{k=1}^{3} \sigma_{k} \frac{\partial}{\partial p_{k}} \text {. }
$$

The differential operators act solely on the power of $p^{2}$ by definition. The numerical constant $c$ is

$$
c=\frac{\pi c_{n j_{1} j_{2}}}{2\left(2 j_{1}+1\right)\left(2 j_{2}+1\right)} 2^{-2 j_{1}-2 j_{2}} \Gamma\left(n-2 j_{1}-2 j_{2}-2\right) \Gamma\left(n-2 j_{1}-2 j_{2}-3\right) .
$$

The next step in simplifying (65) consists in applying the formula

$$
D_{\alpha \beta}^{J}(-\partial)\left(p^{2}\right)^{\mu}=(-2)^{2 J} \frac{\Gamma(\mu+1)}{\Gamma(\mu-2 J+1)} D_{\alpha \beta}^{J}(\hat{P})\left(p^{2}\right)^{\mu-2 J} .
$$

Before one can do this in the non-degenerate case $\left(j_{1} j_{2} \neq 0\right)$, however, we must first reduce both differential operators in (65) by means of vector coupling coefficients

$$
\begin{gathered}
D_{\alpha_{1} \beta_{1}}^{j_{1}}(-\hat{\partial}) D_{\alpha_{2} \beta_{2}}^{j_{2}}(-\hat{\partial})=\sum_{J}\left(j_{1}, \alpha_{1} ; j_{2}, \alpha_{2} \mid J, \alpha_{1}+\alpha_{2}\right) \\
\left(j_{1}, \beta_{1} ; j_{2}, \beta_{2} \mid J, \beta_{1}+\beta_{2}\right) D_{\alpha_{1}+\alpha_{2}, \beta_{1}+\beta_{2}}^{J}(-\hat{\partial}) \square_{p}^{j_{1}+j_{2}-J}
\end{gathered}
$$

and apply the formula

$$
\square_{p}\left(p^{2}\right)^{\lambda}=4 \lambda(\lambda+1)\left(p^{2}\right)^{\lambda-1}
$$

repeatedly. Moreover, it is useful now to perform the substitutions (15) and (20)

and

$$
\begin{gathered}
\Psi(P)=D^{j_{1}}\left(\left(\frac{P}{m}\right)^{\frac{1}{2}}\right) \tilde{\Psi}(P) D^{j_{2}}\left(\left(\frac{P}{m}\right)^{\frac{1}{2}}\right) \\
m=\left(p^{2}\right)^{\frac{1}{2}}>0
\end{gathered}
$$

Under transformations of the homogeneous Lorentz group the field $\tilde{\tilde{\Psi}}_{Q}^{J}(P)$ transforms as in $(21)$, and, if restricted to one mass shell $p^{2}=m^{2}$, 
defines a unitary irreducible representation of the inhomogeneous Lorentz group in the Wigner basis. Performing all the summations gives finally

$$
\begin{aligned}
\|F\|_{n}^{2}= & \sum_{\hat{\imath}=\left|j_{1}-j_{2}\right|}^{j_{1}+j_{2}} \mu_{n j_{1} j_{2}}(\hat{J}) \int_{0}^{\infty} d m^{2}\left(m^{2}\right)^{-d+2} \\
& \cdot \int_{L_{+}} d^{4} p \delta\left(p^{2}-m^{2}\right)\left\{\sum_{Q=-\hat{\imath}}^{+\hat{\jmath}}\left|\tilde{\tilde{\Psi}}_{Q}^{\hat{\jmath}}(P)\right|^{2}\right\}
\end{aligned}
$$

where the spin weight function is

$$
\begin{gathered}
\mu_{n j_{1} J_{2}}(\hat{J})=\frac{\pi c_{n j_{1} j_{2}}}{2\left(2 j_{1}+1\right)\left(2 j_{2}+1\right)} \sum_{J=\left|j_{1}-j_{2}\right|}^{j_{1}+j_{2}}(-1)^{2 j_{1}+2 J_{2}}(2 J+1) \\
\left\{\begin{array}{l}
j_{1} j_{2} J \\
\left.j_{1} j_{2} \hat{J}\right\}
\end{array}\right\} \Gamma\left(n-J-j_{1}-j_{2}-3\right) \Gamma\left(n+J-j_{1}-j_{2}-2\right)
\end{gathered}
$$

with the $6 j$-symbol as defined in Ref. [7].

In the degenerate case one can directly apply (68) to (65) and thus obtain (73), (74) much easier. On the other hand we can simplify (74) and (73) in this case considerably. If, say $j_{2}=0$, then $J=\hat{J}=j_{1}$ and

$$
\left.(-1)^{2 j_{1}+2 j_{2}}(2 J+1)\left\{\begin{array}{l}
j_{1} j_{2} J \\
j_{1}, j_{2}
\end{array}\right)\right\}\left.\right|_{\substack{j_{2}=0 \\
J=\hat{I}=j_{1}}}=1 .
$$

We see that the Hilbert space of distributions $\Psi(P)$ is in fact identical with the Hilbert space of measurable functions $\tilde{\tilde{\Psi}}_{Q}^{J}(P)$ with support on $L_{+}$ that are square integrable in the sense of the norm (73). Let us consider the simplest case $j_{1}=j_{2}=0$. Then

$$
\tilde{\tilde{\Psi}}(P)=\Psi(P)
$$

can be factorized

$$
\Psi(P)=m^{n-2} G(p)
$$

where $G(p)$ is square integrable on $L_{+}$in the Lebesgue sense. This means that for even $n \Phi(x)$ can be represented as the weak derivative of the Fourier transform of $G$

$$
\Phi(x)=\left(-\square_{x}\right)^{\frac{n-2}{2}} \mathscr{F}(G)(x) .
$$

This representation (78) of the field representation $\Phi(x)$ corresponds to $(37)$. 


\section{Extension in $n$}

So far $n$ was restricted by

$$
n>2 j_{1}+2 j_{2}+3
$$

which guarantees that the integral (23) exists for all functions holomorphic inside $\mathbb{D}$, continuous on the boundary. Our aim is to relax this constraint. We want to keep a Hilbert space of holomorphic functions but allow for a different kind of a norm that is obtained by continuation in $n$. For the invariance of the norm under infinitesimal conformal transformations it is necessary that $n$ is real. This analytic continuation is achieved by inserting the value for $c_{n j_{1} j_{2}}$ obtained from (32) with $c_{B}=1$ into (74). This amounts to dividing the norm-squared of a function by the norm-squared of a constant function over $\mathbb{D}$ and guarantees that constant functions remain elements of our Hilbert space during the continuation. The measure $\mu_{n j_{1} j_{2}}(\hat{J})(74)$ thus becomes a meromorphic function of $n$, and we can extend in $n$ from above along the real axis until we reach a first singularity.

We study first the degenerate case $j_{2}=0$

$$
\mu_{n j_{1} 0}\left(j_{1}\right)=\frac{1}{2 \pi^{3}} \Gamma(n) \Gamma\left(n-2 j_{1}-1\right)
$$

that is regular in the half plane

$$
\operatorname{Re} n>2 j_{1}+1 \text {. }
$$

Therefore we have a projective representation of $S U(2,2)$ for any real $n$ satisfying (80) and proper representations for real integers satisfying (80). This way we have obtained two additional representations of the discrete series:

$$
n=2 j_{1}+2 \text { and } n=2 j_{1}+3 .
$$

For the non-degenerate case the singularity appears first in the term $J=j_{1}+j_{2}$. This term involves the factor

$$
\frac{1}{n-2}\left(n-2 j_{1}-2\right)\left(n-2 j_{2}-2\right) \Gamma(n) \Gamma\left(n-2 j_{1}-2 j_{2}-2\right)
$$

that is regular in the half plane

$$
\operatorname{Re} n>2 j_{1}+2 j_{2}+2 \text {. }
$$

Again we have a projective representation for real $n$ in this half plane and proper representations of the discrete series for real integral $n$. Thus we have obtained one further representation of the discrete series

$$
n=2 j_{1}+2 j_{2}+3 \text {. }
$$


The existence of these representations of the discrete series was first asserted by Yao [3]. For the dimension $d(58)$ the corresponding conditions are

$$
\begin{array}{ll}
d>j_{1}+1 & \left(j_{2}=0\right) \\
d>j_{1}+j_{2}+2 & \left(j_{1} j_{2} \neq 0\right) .
\end{array}
$$

It is interesting to compare (82) with the dimensions known in free field theory. For a scalar field $\left(j_{1}=j_{2}=0\right)$ and a spinor field $\left(j_{1}=1 / 2\right.$, $j_{2}=0$ or the other way round) we have the "canonical dimension" $d=1$ respectively $d=3 / 2$. For the electromagnetic current $\left(j_{1}=j_{2}=1 / 2\right)$ and the energy-momentum tensor $\left(j_{1}=j_{2}=1\right)$ we have canonical dimension $d=3$, respectively $d=4$. Thus the canonical dimensions in these four cases appear just as the limiting points of the projective representations, but do themselves not belong to them.

\section{References}

1 Mack, G.. Salam, A.: Ann. Phys 53, 174 (1969)

2. Graev, M L.: Dok1. Akad Nauk SSSR 98, 517 (1954).

3. Yao. T.: J. Math Phys. 8, 1931 (1967), 9, 1615 (1968) The author's statement that "the discrete series were. however, not studied" by Graev is obviously incorrect Yao implements Graev's work quoted on the discrete series only by the few additional cases obtained by extension in $n$, in the non-degenerate case by merely stating its existence

4. Bargmann. V.: Ann. Math 48, 568 (1947).

5. Rüh1. W. Lectures presented at the International Advanced Study Institute on Mathematical Physics in Istanbul, August 1972.

6. Greenberg. O. W.: Ann. Phys 16. 158 (1961).

7. Edmonds, A R. Angular momentum in quantum mechanıcs Princeton University Press 1958.

8. Yosida, K.: Functional analysis. Berlin-Heidelberg-New York: Springer 1968

9. Rühl, W : Commun. math. Phys. 27. 53 (1972).

\section{W. Rühl}

Universität Trier-Kaiserslautern

Fachbereich Physik

D-6750 Kaiserslautern, Pfaffenbergstr 95

Federal Republic of Germany 